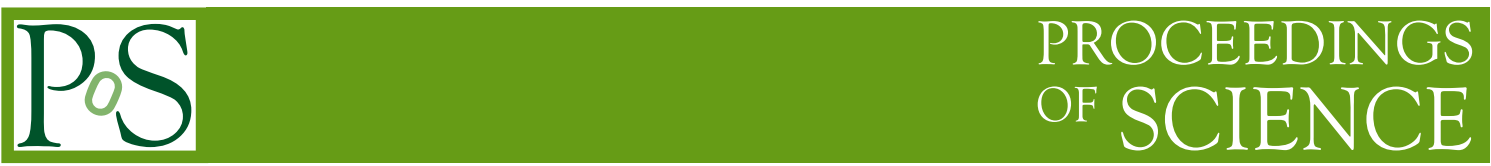

\title{
Recent mass measurements for the $r$ process at JYFLTRAP
}

\author{
A. Kankainen ${ }^{* \dagger}$ T. Eronen; D. Gorelov, J. Hakala, A. Jokinen, V.S. Kolhinen, I.D. \\ Moore, H. Penttilä, S. Rinta-Antila, J. Rissanen, A. Saastamoinen, V. Sonnenschein, \\ and J. Äystö\| \\ P.O. Box 35 (YFL), FI-40014 University of Jyväskylä, Finland \\ E-mail: anu.k.kankainen@jyu.fi
}

\begin{abstract}
This contribution gives an overview of recent mass measurements performed in the ${ }^{132} \mathrm{Sn}$ region relevant for the $r$-process studies. The atomic masses of ${ }^{121-128} \mathrm{Cd},{ }^{129,131} \mathrm{In},{ }^{130-135} \mathrm{Sn}$, ${ }^{131-136} \mathrm{Sb}$, and ${ }^{132-140} \mathrm{Te}$ have been measured with the JYFLTRAP double Penning trap mass spectrometer. Among these, the masses of four $r$-process nuclei $-{ }^{135} \mathrm{Sn},{ }^{136} \mathrm{Sb},{ }^{139} \mathrm{Te}$, and ${ }^{140} \mathrm{Te}$ have been experimentally determined for the first time. In addition, the masses of the $\left(11 / 2^{-}\right)$isomers in ${ }^{121,123,125} \mathrm{Cd}$ and ${ }^{133} \mathrm{Te},\left(1 / 2^{-}\right)$isomers in ${ }^{129,131} \mathrm{In}$, and $\left(7^{-}\right)$isomers in ${ }^{130} \mathrm{Sn}$ and ${ }^{134} \mathrm{Sb}$ have been measured and corresponding excitation energies have been determined. The groundstate mass values have been compared to recent Atomic Mass Evaluations and deviations have been found in particular for the states based on beta-decay energies.
\end{abstract}

XII International Symposium on Nuclei in the Cosmos,

August 5-12, 2012

Cairns, Australia

\footnotetext{
* Speaker.

${ }^{\dagger}$ Present address: University of Edinburgh, Edinburgh, EH9 3JZ, United Kingdom

ॠPresent address: Max-Planck-Institut für Kernphysik, Saupfercheckweg 1, D-69117 Heidelberg, Germany

$\S$ Present address: Lawrence Berkeley National Laboratory, Berkeley, California 94720, USA

IPresent address: Cyclotron Institute, Texas A\&M University, College Station, TX, 77843-3366, USA

" Present address: Helsinki Institute of Physics, P.O. Box 64, FI-00014 University of Helsinki, Finland
} 


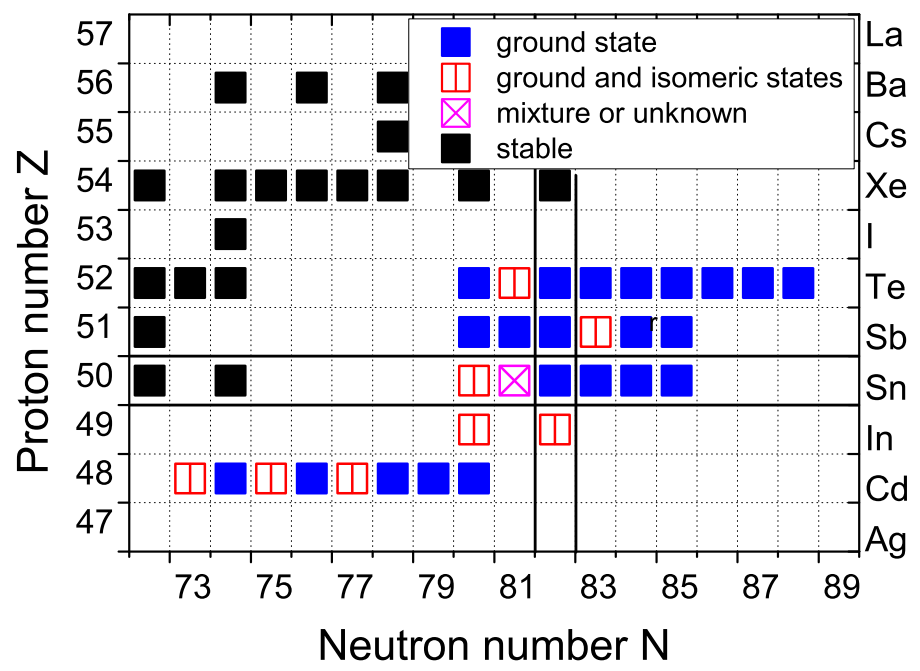

Figure 1: Chart of nuclides showing the measured nuclei and states. A ${ }^{\text {nat }} \mathrm{U}$ target was used to produce neutron-rich ${ }^{121-128} \mathrm{Cd},{ }^{131} \mathrm{In},{ }^{130-135} \mathrm{Sn}$, and ${ }^{132-140} \mathrm{Te}$ in June $2009 .{ }^{129} \mathrm{In}$ and ${ }^{131-136} \mathrm{Sb}$ isotopes were produced with a 14-mg/ $/ \mathrm{cm}^{2}$-thick ${ }^{232} \mathrm{Th}$ target in May 2010.

\section{Introduction}

Exotic nuclei have become accessible for high-precision mass measurements via implementation of Penning trap mass spectrometry at modern radioactive ion beam facilities, see e.g. Ref. [1] for a recent review. The region around ${ }^{132} \mathrm{Sn}$ is interesting to study via mass measurements due to the vicinity of the closed proton $(Z=50)$ and neutron $(N=82)$ shells. In addition, the astrophysical $r$-process $[2,3,4]$ passes through many of the nuclei close to ${ }^{132} \mathrm{Sn}$ on the way towards more neutron-rich nuclei above $Z=50$. Mass measurements provide also data to be compared with different mass models to further develop predictions for experimentally unreachable nuclei. In order to more reliably compare the calculated $r$-process abundances to the observations, the beta-decay properties and masses of the involved nuclei should be precisely known. Due to the $v 1 h_{11 / 2}$ and $\pi 1 g_{9 / 2}$ shells lying close to low- $j$ shells, nuclei close to ${ }^{132} \mathrm{Sn}$ have typically long-living isomeric states. Isomers and low-lying excited states can be thermally populated if the $r$-process operates at high temperatures, and thus, the beta-decay rates can significantly differ from the terrestrial rates [3]. If the $r$-process operates at temperatures so low that thermal equilibrium cannot be achieved, it becomes necessary to independently describe the population of different isomers after neutron capture and the rates for neutron capture and decay of each isomer. We have applied state-of-the-art cleaning methods at the JYFLTRAP double Penning trap mass spectrometer to study the ground states [5] as well as the isomeric states [6] in the ${ }^{132} \mathrm{Sn}$ region (see Fig. 1). The results of these experiments will be discussed in the following sections.

\section{Experimental methods}

The ions of interest were produced via fission reactions induced by $25-\mathrm{MeV}$ protons on a $15-$ $\mathrm{mg} / \mathrm{cm}^{2}$-thick ${ }^{\text {nat }} \mathrm{U}$ or a $14-\mathrm{mg} / \mathrm{cm}^{2}$-thick ${ }^{232} \mathrm{Th}$ target at the Ion Guide Isotope Separator On-Line (IGISOL) facility [7]. The fission products were stopped in the ion-guide gas cell filled with helium 
at a pressure of around 200 mbar. There, a good fraction of ions ended up as singly-charged via charge-exhange reactions. The ions were extracted from the gas cell by differential pumping and with a sextupole ion guide (SPIG) [8]. After acceleration to $30 \mathrm{kV}$ and mass-separation with a $55^{\circ}$ dipole magnet, the continuous ion beam with a selected mass number $A$ was sent to a gas-filled radio-frequency quadrupole cooler and buncher (RFQ) [9]. The RFQ cooled the ions and injected them as narrow ion bunches to the JYFLTRAP double Penning trap [10, 11].

JYFLTRAP consists of two cylindrical Penning traps inside a 7-T superconducting solenoid. The first trap was used for beam purification via a mass-selective buffer-gas cooling technique [12]. This purification trap has a mass resolving power of around $m / \Delta m \approx 3 \times 10^{4}$ which is typically enough to resolve neighbouring isobars but too low for separating isomers. The second trap called precision trap was used for the purification of the beam via Ramsey cleaning technique [13] as well as for the high-precision mass measurements employing the time-of-flight ion cyclotron resonance (TOF-ICR) technique $[14,15]$. With the Ramsey cleaning technique, the isomeric states could be resolved from the ground states (except for ${ }^{131} \mathrm{Sn}$ ), and a mass resolving power up to $m / \Delta m \approx 10^{6}$ was achieved. In the TOF-ICR technique the ion's cyclotron resonance frequency $v_{c}=q B /(2 \pi m)$, where $B$ is the magnetic field, and $q$ and $m$ are the charge and the mass of the ion, is determined by measuring the time-of-flight of the ions from the trap to a microchannel plate detector (MCP). After magnetron and quadrupole excitations in the precision trap, the ions excited under resonance conditions gain more radial energy and are accelerated by a stronger axial force in the magnetic field gradient when extracted from the trap towards the MCP outside the solenoid. Thus, the ions in the resonance have the shortest time-of-flights. Figure 2 shows an example of a TOF-ICR spectrum for ${ }^{138} \mathrm{Te}^{+}$ions. The magnetic field $B$ was calibrated with Xe ions with well-known masses $m_{\text {ref }}$ : ${ }^{130} \mathrm{Xe}(m=129.903509351(15) \mathrm{u}[16]),{ }^{132} \mathrm{Xe}(m=131.904155086(10) \mathrm{u}[17])$ and ${ }^{134} \mathrm{Xe}(m=$ 133.9053945(9) u [18]). The mass of the nuclide of interest was determined for these singlycharged ions as:

$$
m_{\text {meas }}=r\left(m_{r e f}-m_{e}\right)+m_{e},
$$

where $r=\frac{v_{c, r e f}}{v_{c, \text { meas }}}$ is the cyclotron frequency ratio between the reference ion and the ion of interest, and $m_{e}$ is the electron mass.

To reduce the uncertainty due to time-dependent fluctuations in the magnetic field, the data were collected interleavedly [19]: after one or two frequency sweeps for the reference ion, a few frequency sweeps were collected for the ion of interest and this pattern was repeated as long as required for sufficient statistics. The collected data files were split into smaller parts in such a way that a proper count-rate class analysis [20] was possible at least for the reference ion. The error due to the time-dependent magnetic field fluctuation, $\delta_{B}\left(v_{r e f}\right) / v_{r e f}=5.7(8) \times 10^{-11} \mathrm{~min}^{-1} \Delta \mathrm{t}$, where $\Delta t$ is the time between the two reference measurements, was quadratically added to the statistical uncertainty of each frequency ratio. The weighted mean of the measured frequency ratios was calculated and used as the final value. The inner and outer errors [21] of the data sets were compared and the larger value of these two was taken as the error of the mean. Finally, the uncertainty due to the mass-dependent shift $\delta_{m}(r) / r=\left(7.5 \pm 0.4 \times 10^{-10} / u\right) \times \Delta m$ [22] and an additional residual relative uncertainty $\delta_{\text {res }}(r) / r=7.9 \times 10^{-9}$ [22] were quadratically added to the error. 


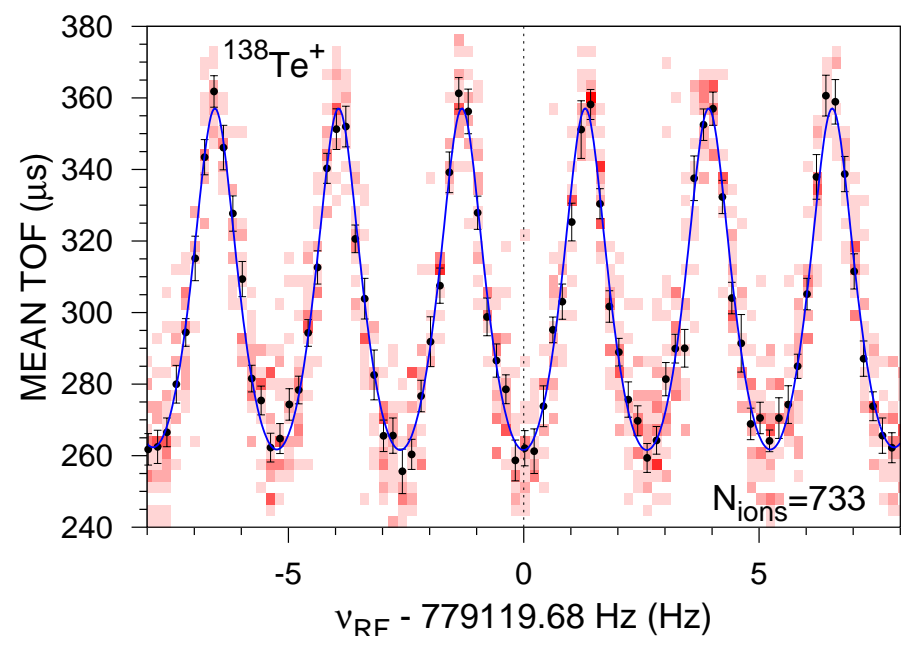

Figure 2: A TOF-ICR spectrum of ${ }^{138} \mathrm{Te}^{+}$obtained with an excitation pattern of $25 \mathrm{~ms}$ (On) - $350 \mathrm{~ms}$ (Off) - $25 \mathrm{~ms}(\mathrm{On})$ fitted with the theoretical lineshape for time-separated oscillatory fields [23, 24]. The number of ions in a bunch has been limited to $1-2$ ions/bunch and the time gate has been set to $224-384 \mu s$ for this figure. The red squares indicate the number of ions in each time-of-flight bin: the darker the color, the more ions there are. The dashed line shows the position of the resonance frequency.

\section{Ground-state masses}

The JYFLTRAP results [5] have already been included in the latest Atomic Mass Evaluation (AME12) [25]. In order to show the impact of the new mass measurements, differences between the JYFLTRAP values and the previous evaluation from 2003 (AME03) [18] as well as with the preview of the AME12, called here as AME11 [26], have been plotted for the measured Cd, Sn, $\mathrm{Sb}$ and Te ground states in Fig. 3. Two important trends can be seen in Fig. 3: the more neutronrich isotopes tend to deviate more from the AME values and the extrapolated values of the more recent evaluation (AME11) agree with JYFLTRAP better than the values of the AME03. The biggest deviation between the extrapolated and JYFLTRAP values has been decreased from around $600 \mathrm{keV}$ (AME03) to around $240 \mathrm{keV}$ (AME11). For ${ }^{129} \mathrm{In}$ and ${ }^{131} \mathrm{In}$ not plotted in Fig. 3, the deviations were decreased from about $100 \mathrm{keV}$ (AME03) and to about $24 \mathrm{keV}$ (AME11) and a better agreement was obtained with the latest AME values.

The most striking differences between the ground-state mass excesses and the AME11 values are found for ${ }^{123} \mathrm{Cd},{ }^{132,134,135} \mathrm{Sb}$, and ${ }^{134-136} \mathrm{Te}$ (see Fig. 3). The case of ${ }^{123} \mathrm{Cd}$ will be discussed in section 4. The discrepancies between the AME11 values and JYFLTRAP for ${ }^{132,134,135} \mathrm{Sb}$, and ${ }^{134-136} \mathrm{Te}$ can be explained by biased beta-decay data $[27,28,29]$ used for the evaluation. These beta-decay experiments give systematically too low mass-excess values compared to JYFLTRAP. Recent precision mass measurements on ${ }^{132} \mathrm{Sb}$ and ${ }^{134-136} \mathrm{Te}$ at the Canadian Penning trap (CPT) [30] agree well with JYFLTRAP and their value for ${ }^{134} \mathrm{Sb}$ is in agreement with the JYFLTRAP value for the isomeric state. The value from an experiment on ${ }^{135} \mathrm{Sb}$ in the experimental storage ring (ESR) at the fragment separator (FRS) [31] agrees with the JYFLTRAP value. In general, the ISOLTRAP data $[32,33]$ support the JYFLTRAP results. These independent measurements have given further support that some of the old AME values should be revised in this region. 

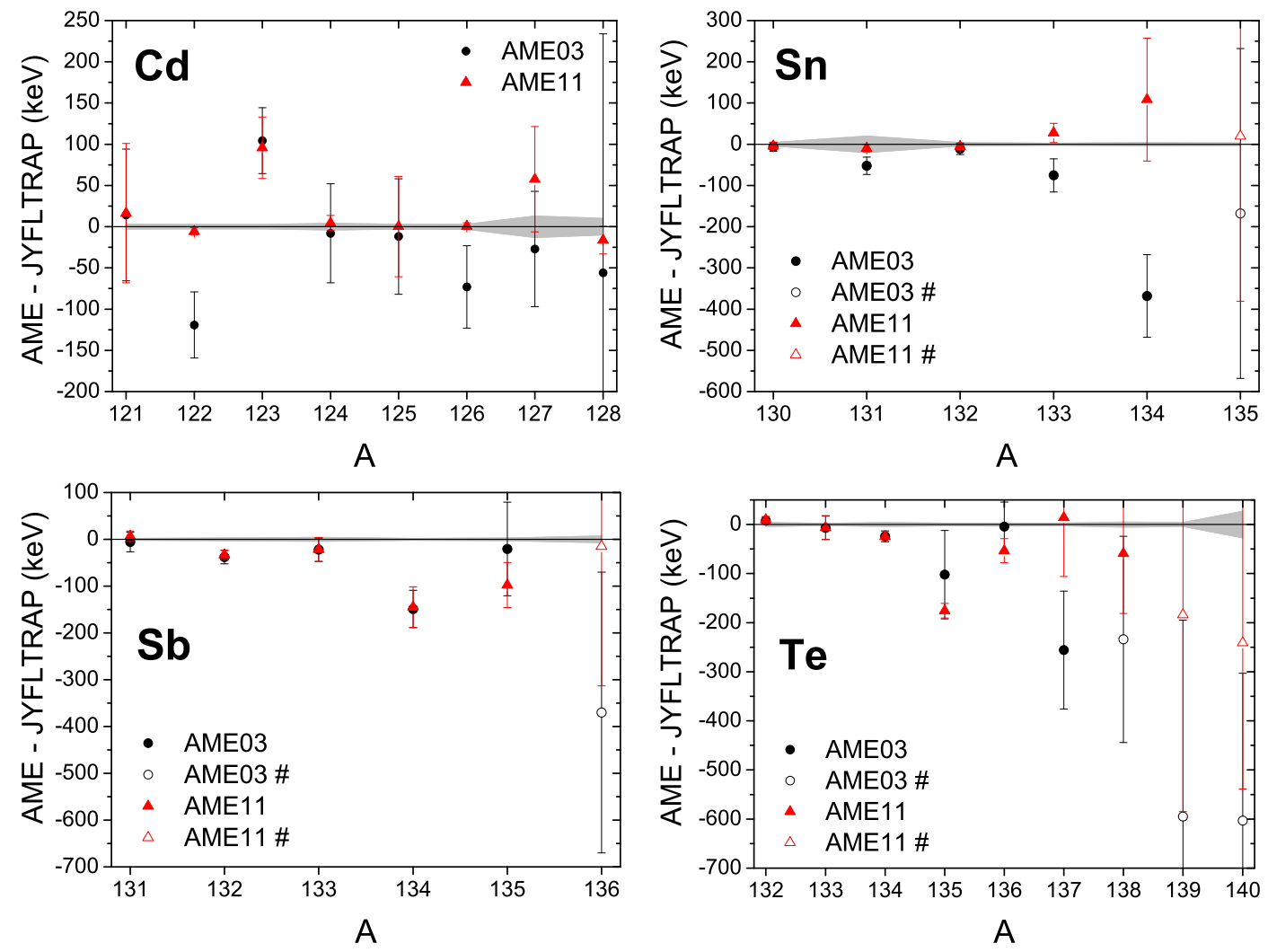

Figure 3: Comparison between the JYFLTRAP results and Atomic Mass Evaluations from 2003 (AME03) [18] and 2011 (preview) [26]. The gray-shaded region shows the error band for the JYFLTRAP results whereas the data points show the AME values with respect to JYFLTRAP values. The uncertainties of the data points include only errors from the AME03 and AME11. The AME12 [25] was not used for comparison since the JYFLTRAP data have already been included in it.

\section{Excitation energies for isomers}

By using the dipolar cleaning technique, isomers could be resolved from the ground states except for ${ }^{131} \mathrm{Sn}$. For ${ }^{131} \mathrm{Sn}$, the isomeric state is expected to be at $65.1 \mathrm{keV}$ [34] which is too low to be resolved with JYFLTRAP at the moment. The excitation energies for the $\left(11 / 2^{-}\right)$isomer in ${ }^{125} \mathrm{Cd}$ and $\left(1 / 2^{-}\right)$isomers in ${ }^{129,131}$ In have previously been determined via beta-decay energy differences, and therefore, JYFLTRAP results improved the precisions significantly. A good agreement was found between the JYFLTRAP results [6] and the precise excitation energies based on $\gamma$-ray energies for ${ }^{121} \mathrm{Cd}\left(11 / 2^{-}\right),{ }^{130} \mathrm{Sn}\left(7^{-}\right)$and ${ }^{134} \mathrm{Sb}\left(7^{-}\right)$isomers.

Figure 4 shows the trend of the excitation energies of the $11 / 2^{-}$isomers as a function of neutron number $N$ for odd- $N$ Cd, Sn, Te and Xe isotopes. The JYFLTRAP results for Cd isotopes are plotted as red squares connected by a thick red line. The trend is totally different from the previously adopted values shown as open squares connected by a dashed red line. However, it follows well the trend of the Te isotopes which have two protons above the closed $Z=50$ shell instead of the two proton holes for the Cd isotopes. The value for ${ }^{125} \mathrm{Cd}^{m}$ differs from the result based on beta decay [35] but as shown with the ground-state masses (see section 3), the beta- 


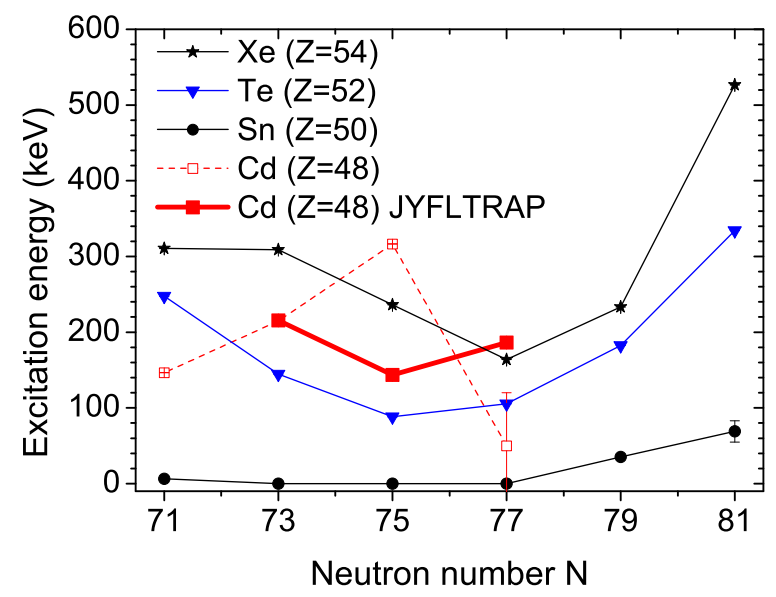

Figure 4: Excitation energies of $11 / 2^{-}$states of odd- $N$ isotopes close to ${ }^{132} \mathrm{Sn}$.

decay energies are often biased e.g. due to unobserved feeding to higher-lying states [36]. The excitation energy of the isomer in ${ }^{123} \mathrm{Cd}, 316.52(23) \mathrm{keV}$ [37], is not based on observations of $\gamma-\gamma$ coincidences or on a direct measurement of $\gamma$-transition energy. It was obtained from a search where the number of shared levels de-exciting to the ground and isomeric states was maximized. Two shared levels were found when a $\gamma$-ray energy range of $260-400 \mathrm{keV}$ was applied for the search. The JYFLTRAP result is below this energy range and disagrees strongly with Ref. [37]. Based on the JYFLTRAP result, the most probable shared level would be the state at $263.87(2) \mathrm{keV}$ [37] de-excited by $263.87(2) \mathrm{keV}$ and $123.67(6) \mathrm{keV} \gamma$-transitions to the ground and isomeric states, respectively. The beta decay of ${ }^{123} \mathrm{Ag}$ should be reinvestigated to revise the decay scheme. The mass of ${ }^{123} \mathrm{Cd}$ has also been studied at ISOLTRAP but the ground and isomeric states could not be resolved there [33].

\section{Discussion}

The ground-state mass values have been used for studies of odd-even staggering of binding energies and related empirical pairing gaps across the $N=82$ shell gap [5] by using the threepoint odd-even-mass-staggering formula [38]: $\Delta^{(3)}(N)=(-1)^{N}[M E(N+1)-2 M E(N)+M E(N-$ $1)] / 2$, where $M E$ is the corresponding mass excess. The odd-even staggering $\Delta^{(3)}(N)$ depends on the intensity of pairing correlations in nuclei but is also affected by the polarization effects. Figure 5 shows both the experimental results and theoretical calculations on the staggering over the $N=82$ closed neutron shell. Whereas the theoretical calculations yield very similar results for the staggering of the odd- $N$ isotopes of $\mathrm{Sn}, \mathrm{Te}$, and Xe across the $N=82$ shell, the trend in experimental values is different. The neutron pairing gap for $\mathrm{Sn}$ is decreased by about $460 \mathrm{keV}$ from $N=81$ to $N=83$ (in agreement with theory) but for Te or Xe isotopes this reduction is much smaller, about $170 \mathrm{keV}$ for Te and only about $40 \mathrm{keV}$ for Xe. In Ref. [5], several theoretical models (spherical HFB, deformed HFB, deformed particle-number conserving and self-consistent calculations) were tried but the staggering values of the $N=83$ isotones as a function of the proton number $Z$ remained a puzzle. 

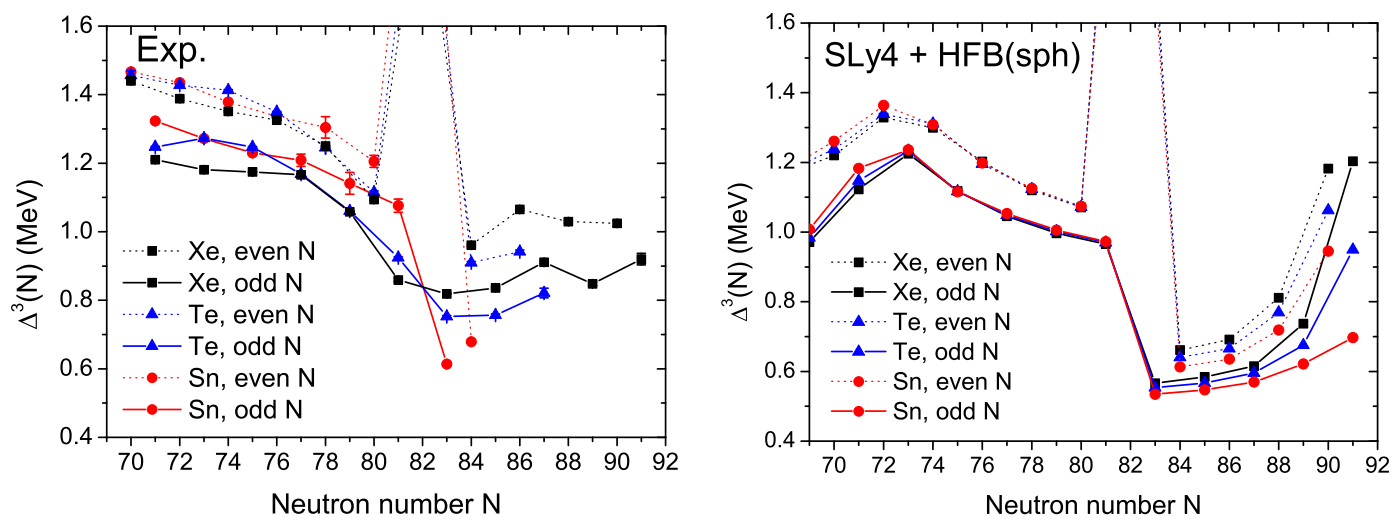

Figure 5: The odd-even staggering around the $N=82$ closed neutron shell based on experiments (left) and on theoretical calculations employing Sly4 [39] energy density functional combined with spherical HartreeFock-Bogoliubov (HFB) approximations (right). Whereas the steep decrease in the experimental staggering values for the odd- $N \mathrm{Sn}$ isotopes across the $N=82$ shell closure is rather well produced by the theoretical calculations, the flattened trend above the $Z=50$ seen in the odd- $N$ isotopes of Te and Xe cannot be explained by current models. For details, see Ref. [5].

As a summary, exotic radioactive isotopes in the ${ }^{132} \mathrm{Sn}$ region have been produced via protoninduced fission reactions at the IGISOL facility. The JYFLTRAP double Penning trap mass spectrometer has been used to resolve long-lived (>100 ms) isomeric and ground states from each other and to measure their masses [5, 6] with a typical precision of about $\delta m / m \approx 10^{-8}$. The measurements have served results with superior precision compared to older beta-decay studies which have had a tendency to underestimate the mass values. Since it has been possible to determine excitation energies for the isomers with JYFLTRAP, useful information on single-particle energies e.g. for $(11 / 2-)$ states in odd $\mathrm{Cd}$ isotopes and $\left(1 / 2^{-}\right)$states in In isotopes have been obtained. Many of the measured nuclei are located at the $r$-process path, and therefore, their masses are crucial for an accurate modeling of the process. The JYFLTRAP measurements have shown that the AME03 values deviate from the measured values up to around $600 \mathrm{keV}$. The more recent evaluation, AME11, shows smaller differences to the JYFLTRAP results, but still, deviations can be up to around 240 $\mathrm{keV}$. It is also worthwhile to note that for example the value for ${ }^{135} \mathrm{Te}$ in the AME11 [26] deviates from the recent Penning trap measurements at JYFLTRAP [5] and CPT [30] by about $170 \mathrm{keV}$ (more than 9 $\sigma$ ). The effect of these new measurements on the AME12 [25] has been significant. The high-precision mass measurements also serve data for mass model developments, which are needed for experimentally unreachable $r$-process nuclei. The analysis on the impact of these results on the $r$-process calculations is ongoing.

\section{Acknowledgments}

This work has been supported by the Academy of Finland under the Finnish Centre of Excellence Programme 2006-2011 (Nuclear and Accelerator Based Physics Programme at JYFL). A.K. acknowledges the support from the Academy of Finland under the project 127301. 


\section{References}

[1] K. Blaum, J. Dilling, and W. Nörtershäuser, Phys. Scr. 2013, 014017 (2013).

[2] E. M. Burbidge, G. R. Burbidge, W. A. Fowler, and F. Hoyle, Rev. Mod. Phys. 29, 547 (1957).

[3] M. Arnould, S. Goriely, and K. Takahashi, Phys. Rep. 450, 97 (2007).

[4] A. Arcones and G. Martínez-Pinedo, Phys. Rev. C 83, 045809 (2011).

[5] J. Hakala et al., Phys. Rev. Lett. 109, 032501 (2012).

[6] A. Kankainen et al., Phys. Rev. C 87, 024307 (2013).

[7] J. Äystö, Nucl. Phys. A 693, 477 (2001).

[8] P. Karvonen et al., Nucl. Instrum. Methods Phys. Res. B 266, 4794 (2008).

[9] A. Nieminen et al., Nucl. Instrum. Methods Phys. Res. A 469, 244 (2001).

[10] V. S. Kolhinen et al., Nucl. Instrum. Methods Phys. Res. A 528, 776 (2004).

[11] T. Eronen et al., Eur. Phys. J. A 48, 46 (2012).

[12] G. Savard et al., Phys. Lett. A 158, 247 (1991).

[13] T. Eronen et al., Nucl. Instrum. Methods Phys. Res. B 266, 4527 (2008).

[14] G. Gräff, H. Kalinowsky, and J. Traut, Z. Phys. A 297, 35 (1980).

[15] M. König et al., Int. J. Mass Spectrom. Ion Processes 142, 95 (1995).

[16] M. Redshaw, B. J. Mount, E. G. Myers, and F. T. Avignone, Phys. Rev. Lett. 102, 212502 (2009).

[17] M. Redshaw, B. J. Mount, and E. G. Myers, Phys. Rev. A 79, 012506 (2009).

[18] G. Audi, A. H. Wapstra, and C. Thibault, Nucl. Phys. A 729, 337 (2003).

[19] T. Eronen et al., Phys. Rev. Lett. 103, 252501 (2009).

[20] A. Kellerbauer et al., Eur. Phys. J. D 22, 53 (2003).

[21] R. T. Birge, Phys. Rev. 40, 207 (1932).

[22] V.-V. Elomaa et al., Nucl. Instrum. Methods Phys. Res. A 612, 97 (2009).

[23] M. Kretzschmar, Int. J. Mass Spectrom. 264, 122 (2007).

[24] S. George et al., Int. J. Mass Spectrom. 264, 110 (2007).

[25] M. Wang et al., Chin. Phys. C 36, 1603 (2012).

[26] G. Audi and M. Wang, Private Communication, April 2011, by Georges Audi and Wang Meng (unpublished).

[27] P. Hoff, B. Ekström, and B. Fogelberg, Z. Phys. A 332, 407 (1989).

[28] B. Fogelberg et al., Phys. Rev. Lett. 82, 1823 (1999).

[29] B. Fogelberg et al., Phys. Rev. C 75, 054308 (2007).

[30] J. Van Schelt et al., Phys. Rev. C 85, 045805 (2012).

[31] B. Sun et al., Nucl. Phys. A 812, 1 (2008).

[32] M. Dworschak et al., Phys. Rev. Lett. 100, 072501 (2008). 
[33] M. Breitenfeldt et al., Phys. Rev. C 81, 034313 (2010).

[34] B. Fogelberg et al., Phys. Rev. C 70, 034312 (2004).

[35] H. Huck et al., Phys. Rev. C 39, 997 (1989).

[36] J. C. Hardy, L. C. Carraz, B. Jonson, and P. G. Hansen, Phys. Lett. B 71, 307 (1977).

[37] H. Huck et al., Phys. Rev. C 40, 1384 (1989).

[38] W. Satuła, J. Dobaczewski, and W. Nazarewicz, Phys. Rev. Lett. 81, 3599 (1998).

[39] E. Chabanat et al., Nucl. Phys. A 635, 231 (1998). 